\title{
Discours métaphorique et monnaies : les particularités de l'euro
}

Catherine Resche

\section{(2) OpenEdition}

\section{Journals}

Édition électronique

URL : http://journals.openedition.org/asp/2754

DOI : 10.4000/asp. 2754

ISSN : 2108-6354

\section{Éditeur}

Groupe d'étude et de recherche en anglais de spécialité

\section{Édition imprimée}

Date de publication : 1 décembre 1998

Pagination : 67-88

ISSN : 1246-8185

\section{Référence électronique}

Catherine Resche, « Discours métaphorique et monnaies : les particularités de l'euro », ASp [En ligne] 19-22 | 1998, mis en ligne le 02 mars 2012, consulté le 19 avril 2019. URL : http:// journals.openedition.org/asp/2754 ; DOI : 10.4000/asp.2754

Ce document a été généré automatiquement le 19 avril 2019

Tous droits réservés 


\title{
Discours métaphorique et monnaies : les particularités de l'euro
}

\author{
Catherine Resche
}

1 Cette étude est le prolongement d'un article sur l'activité néologique de la langue (Resche 1998) où nous avons ébauché l'hypothèse selon laquelle une analyse des néologismes formés autour de « euro » (compris à la fois au sens de l'Union européenne et au sens de monnaie unique) permettait de témoigner de l'évolution des idées pendant une période donnée en se faisant l'écho des diverses prises de position face aux enjeux importants pour tous les citoyens concernés.

2 Nous voudrions maintenant concentrer notre investigation sur cet aspect particulier de la néologie qu'est la métaphore et nous intéresser au message que peut laisser entrevoir une étude plus poussée du discours métaphorique de l'euro, monnaie unique. Pour ce faire, nous nous appuierons sur différentes théories de la métaphore et sur les approches diverses qu'elles ont suscitées; puis, nous examinerons les registres métaphoriques communs à diverses monnaies et à l'euro afin de faire ressortir la spécificité des métaphores qui est propre à la monnaie unique, ce qui devrait nous permettre d'ouvrir quelques pistes quant aux fonctions du discours métaphorique et au contenu du message véhiculé.

\section{Les problèmes théoriques posés par la métaphore}

\subsection{Métaphore-mot ou métaphore-énoncé : débat entre sémantique et sémiotique}

3 La définition qu'Aristote a donnée de la métaphore a influencé la pensée occidentale pendant des siècles. Selon la Poétique, (1457 b: 6-9), 
la métaphore est le transport à une chose d'un nom qui en désigne une autre, transport ou du genre à l'espèce, ou de l'espèce au genre ou de l'espèce à l'espèce ou d'après le rapport d'analyse.

Comme le résume très clairement Paul Ricœur dans sa préface,

La rhétorique de la métaphore prend le mot pour unité de référence. La métaphore, en conséquence, est classée parmi les figures de discours en un seul mot et définie comme trope par ressemblance; en tant que figure, elle consiste dans un déplacement et dans une extension du sens des mots; son explication relève d'une théorie de la substitution. (1975:7)

Dans l'analyse très riche qu'il fait des théories successives et concurrentes de la métaphore, Paul Ricœur souligne bien que si le point de vue sémantique, qui replace la métaphore dans le cadre de la phrase, semble s'opposer à la notion de métaphore-mot d'Aristote, le mot ne doit pas pour autant être complètement éliminé : reprenant la terminologie de Max Black, Ricœur précise que « le mot reste le 'foyer', même s'il requiert le 'cadre' de la phrase ». Et, ajoute-t-il, si le mot reste le support de sens métaphorique, c'est parce que, dans le discours, la fonction du mot est d'incarner l'identité sémantique. Or, c'est cette identité sémantique que la métaphore transforme. Il ne faut pas oublier que le sens métaphorique n'est pas inhérent au mot mais qu'il ressort de la combinaison et de l'interaction des mots dans la phrase, d'où la nécessité de prendre en compte le syntagme dans l'énoncé métaphorique, ce que nous ferons puisque nous partirons des unités simples ou composées pour aller jusqu'à la phrase, ou un ensemble de phrases dans le cas de la métaphore filée.

\subsection{Métaphore lexicalisée et métaphore nouvelle}

5 Si, pour l'auteur de la Poétique, la métaphore est essentiellement un effet de style, un écart par rapport à la langue ordinaire, on peut supposer, à la relecture d'un court passage du livre 3 de la Rhétorique cité par Jean Tournier (1985), qu'Aristote a été conscient que, de manière courante, chacun parle par métaphores, sans pour autant s'en rendre compte. Ricœur citant Boileau et Dumarsais à ce propos, relève que :

Il s'en fait plus aux halles en un jour de marché qu'il n'y en a dans toute l'Enéide, ou qu'il ne s'en fait à l'Académie dans plusieurs séances consécutives...

On les sait par usage, comme la langue maternelle, sans qu'on puisse dire quand et comment on les a apprises. (1975:85),

6 De fait, en appliquant ces remarques à la langue économique, sans entrer encore dans le domaine plus pointu des monnaies qui nous intéresse ici, nous pouvons citer un bon nombre de termes et de phraséologismes qui relèvent de métaphores lexicalisées.

7 Il suffit de considérer des termes aussi courants que "inflation", "deflation », "ballooning financial markets», "a bubble economy», ou la phrase: "the bubble is about to burst » pour se rendre à l'évidence. De la même façon, « financial instruments » ou « economic tools» ne seront pas immédiatement identifiés à la métaphore sousjacente. Le lecteur ou le locuteur penseront-ils à une machine ou un moteur en parlant de la « surchauffe » de l'économie (" an overheating economy ») ? Auront-ils conscience du double sens métaphorique (personnification / santé) induit par des expression telles que " a healthy economy », « an ailing sector ", « recovery », « economic health»? Que dire encore du registre nautique à l'origine de "floating rates", "an illiquid market", « emerging economies », etc. ? 
Il est évident qu'avec l'usure de la métaphore par la banalisation de l'emploi, le phénomène de transfert de sens est oublié; on est véritablement en présence d'un nouveau sens et l'on peut penser qu'une étude de ce processus de métaphorisation sur une plus longue durée pourrait s'avérer riche d'enseignement quant à l'évolution des mentalités et révélatrice au plan culturel. Pour Le Guern (1978), ce processus joue un rôle considérable dans la création et l'évolution du vocabulaire, puisque

une part importante des mots dont nous nous servons est constituée par l'ensemble des apports successifs fournis par la lexicalisation des métaphores.

Qu'en est-il alors de la métaphore "d'invention»? Quelle définition en donner d'ailleurs? Doit-on restreindre cette notion à la période relativement courte pendant laquelle un auteur, quel qu'il soit, aura véritablement innové en percevant ou en créant une ressemblance inattendue, ignorée jusqu'alors? Doit-on penser qu'une fois cette métaphore reprise par d'autres, elle entre dans le registre commun, dans le fond de la langue et meurt en tant que telle? En avançant qu'une des fonctions de la métaphore est de combler un vide sémantique, Aristote pourrait offrir une réponse. Sous cet angle, effectivement, la métaphore d'invention prend tout son sens, puisqu'elle permet d'exprimer une notion pour laquelle il n'existe pas de signifié dans la langue. Mais si cette approche se comprend pour la conception de la métaphore-mot, et en partie seulement, elle est trop restreinte pour ce qui est de la métaphore-discours. On en revient alors au traitement sémantique de la métaphore où la phrase est considérée comme première unité de signification et où la métaphore d'invention se conçoit comme une « attribution insolite au niveau du discours-phrase » (Ricœur, 1975).

Pour notre part, dans la mesure où nous travaillons sur une langue spécialisée, et non sur la langue littéraire ou poétique, nous ne parlerons pas de métaphores d'invention mais de «métaphores d'extension », comme nous l'expliquerons par la suite.

\subsection{Fonctions de la métaphore}

\subsubsection{Fonction rhétorique}

11 Avant même la rhétorique d'Aristote, qui couvrait les trois domaines de l'argumentation, de l'élocution et de la composition du discours, il y avait tout simplement l'éloquence, c'est-à-dire la parole considérée comme moyen d'influencer l'autre, à quelque niveau que ce soit, pouvoir redoutable, on le conçoit, puisque la maîtrise de la parole peut être mise à profit pour jouer avec les mots et, par ce biais, manipuler les hommes. A ce sujet, il sera intéressant de voir si le discours métaphorique de l'euro est caractérisé par ce que l'on pourrait appeler un retour à la rhétorique originelle, à savoir un moyen et une intention d'influencer l'autre, ici les peuples, les sceptiques, etc.

\subsubsection{Fonction poétique, ornementale}

12 La métaphore est très souvent étudiée dans la perspective de la poésie où sa fonction n'est pas la persuasion, mais une transposition artistique de ce qui pourrait être dit plus ordinairement, de manière courante. Dans son De Oratore, Cicéron explique que la métaphore a pour rôle de procurer plaisir et agrément, et de faire en sorte que l'expression gagne en couleur. Si l'on applique cette idée au domaine plus prosaïque de la finance, par exemple, la question du choix de substituer un mot métaphorique à un autre mot existant relève peut-être de ce souci esthétique. Mais, quand «shark» vient prendre 
la place de «swindler», peut-on dire que la métaphore n'a qu'une valeur décorative ? Pourquoi utiliser "shark», si l'on peut se satisfaire de "swindler»? N'y a-t-il pas au contraire une autre fonction de la métaphore à envisager? On pense évidemment à la fonction rhétorique : au travers de la connotation négative par laquelle le locuteur ou l'auteur insistent davantage sur le sème commun aux requins et aux escrocs de la finance, à savoir la voracité (Tournier 1993 : 119), ils se livrent à une retranscription de la réalité et cherchent ainsi à influencer leur public.

\subsubsection{Fonction « lexicale » de la métaphore}

13 N'oublions pas que, comme nous l'avons mentionné, Aristote a envisagé la métaphore comme moyen de combler une lacune sémantique, ce qui suffit à prouver que la métaphore ne peut être réduite, dans tous les cas, à un simple ornement. En permettant d'exprimer un fait, de parler d'une chose, alors que les mots pour le faire n'existent pas dans la langue, elle décrit la réalité et aide à informer. Pour retourner à Cicéron (De Oratore), nous rappellerons que, sans la possibilité ouverte par la métaphore, la langue n'aurait pas assez de ressources pour répondre à tous les besoins de communication. C'est ainsi que, dans le souci d'éviter de longues périphrases, lorsque l'on veut décrire une personne qui pratique plutôt une politique de changements en douceur, on aura recours à "dove ", par analogie avec la douceur qui caractérise la colombe. A contrario, "hawk" fera allusion à celui qui adopte ou prône des mesures draconiennes, qui réagit en sortant ses griffes comme le faucon pour fondre sur sa proie. Pour preuve, bon nombre d'articles portant sur la politique monétaire menée par la Réserve Fédérale américaine qualifient Alan Greenspan de "dovish" pour décrire son flegme devant ce que d'autres, plus agressifs, interprètent comme une menace d'inflation qui justifierait, à leurs yeux, des réactions plus radicales.

\subsubsection{Fonction heuristique de la métaphore}

14 Si Aristote lui-même a pu écrire (Poétique, 1459 a 4-8, cité par Ricœur, 1975) que « bien faire les métaphores, c'est bien apercevoir les ressemblances ", ne peut-on pas aller jusqu'à ajouter que c'est aussi les faire entrevoir aux autres, en attirant leur attention, en traduisant ce qui peut paraître compliqué en termes plus imagés qui trouvent un écho dans l'expérience de tout un chacun? Quand on donne à l'abstrait l'aspect du concret, quand on parle de ce qui est nouveau en utilisant le langage de ce qui est connu, quand on présente à un apprenant une technique qui ne lui est pas familière en ayant recours à des métaphores, on cherche à souligner les points communs avec des situations aisément compréhensibles pour le public. Ainsi, pour introduire le traitement de texte à des néophytes, on choisira un discours qui empruntera au registre d'un plan de ville ou d'un cabinet de médecin (Corbisier 1994: 50-51) pour faire passer le message et faciliter l'apprentissage. On en arrive ainsi à parler de l'aspect iconique de la métaphore qui crée une image mentale dans l'esprit du récepteur. C'est ce qui lui donne une fonction didactique.

\subsubsection{La métaphore comme moyen d'expression euphémique}

Enfin, nous avons tous fait l'expérience de la métaphore comme moyen de minimiser l'impact d'une assertion. L'exemple le plus parlant en est la variété de termes métaphoriques pour évoquer la mort, communément décrite comme un départ, un 
voyage, le repos ou le sommeil, ce qui a donné lieu à des euphémismes du type « to depart ", « to pass away », « his/her last journey », ou encore " eternal rest » (Tournier 1993 : 135).

Dans l'analyse qui va suivre, il conviendra de déterminer quelles fonctions de la métaphore sont mises en œuvre dans les exemples que nous examinerons. À la lumière de ces quelques remarques, nous allons maintenant concentrer notre étude sur les métaphores communes aux monnaies en général, avant d'envisager la particularité de l'euro et de tirer un enseignement de ces différences.

\section{Monnaies et métaphores}

\subsection{Le corpus}

17 La question qui s'est posée à nous était de décider si nous devions restreindre notre corpus à la presse économique et financière spécialisée ou l'élargir à d'autres sources moins spécialisées, mais s'intéressant au domaine de la finance et des monnaies entre autres. Nous avons opté pour le second choix, pour la simple raison que nous risquions d'exclure la possibilité d'étudier certaines des fonctions de la métaphore mentionnées cidessus en nous concentrant exclusivement sur des sources très pointues dans ce domaine. De plus, les quotidiens ou hebdomadaires, spécialisés ou non, se font aussi bien l'écho des hommes politiques, des économistes ou professeurs d'université spécialistes du sujet que des journalistes, chroniqueurs ou éditorialistes et il nous a semblé qu'il serait intéressant, précisément, de comparer les différents niveaux de discours et de publics cibles. Nous avons donc retenu les sources suivantes: The Wall Street Journal, Financial Times, The Economist, Business Week, Newsweek, et Time, ainsi que des documents diffusés par les banques centrales ou autres banques. Il nous reste à préciser que nous n'avons fondé notre analyse que sur les registres métaphoriques communs à ces différentes sources car nous voulions éviter de prendre en compte des styles très ponctuels qui ne seraient pas révélateurs d'une tendance générale et dont nous ne pourrions tirer des conclusions fiables.

\subsection{Métaphores lexicalisées}

Il va de soi que le registre des monnaies n'échappe pas au phénomène que nous avons déjà mentionné, c'est-à-dire qu'on y trouve de nombreux exemples de métaphores lexicalisées que spécialistes et commentateurs reprennent naturellement et dont on peut dire qu'elles font désormais partie du fond de la langue pour ce domaine précis.

Parmi ces métaphores figées, nous citerons aussi bien des noms, des adjectifs que des verbes. Ainsi, "flows of capital », " currency fluctuations ", « a choppy market ", " anchor currency ", " to plunge, to dive, to drift, to sink » sont totalement banalisés aujourd'hui. Il en est de même pour d'autres termes ordinairement associés aux monnaies et qui ne retiennent plus l'attention au quotidien par leur connotation métaphorique. On utilisera machinalement "money in circulation», "Exchange Rate Mechanism», "to attack a currency ", " to defend a currency ", " a weaker yen ", " a stronger dollar ", sans penser immédiatement aux registres d'emprunt, à savoir la mécanique ou les véhicules, la guerre ou le combat et sans prendre conscience du processus de personnification. De fait, les termes mis en relief constituent des collocations usuelles dès lors que l'on parle des monnaies et leur sens figuré semble avoir totalement disparu. 

juillet 1998: 80, « The Winners in a Losing Year ») :

Just when it seemed the worst was over, economic crises in Russia and Japan have investors wondering if emerging markets will stay submerged indefinitely.

La question qui se pose ici est de savoir si la présence de «submerged» dans le contexte immédiat de « emerging markets » ne conduit pas à jeter une nouvelle lumière sur ce terme et, en quelque sorte, à raviver ce que l'on pourrait considérer comme une métaphore morte, et que nous qualifierons, dans ce cas, de métaphore en sommeil.

Dans cette optique, nous avons procédé à un recensement dans notre corpus des métaphores au niveau de la phrase, et non plus du mot, et nous avons remarqué que les champs métaphoriques ainsi couverts correspondaient aux registres d'origine des métaphores lexicalisées citées plus haut en exemple. Autrement dit, tout se passe comme si ces métaphores banalisées ouvraient la voie à des métaphores nouvelles, de telle sorte que nous pouvons considérer les premières comme des métaphores-souches, et les dernières comme des métaphores d'extension. Les métaphores-souches serviraient alors d'archétypes pour des métaphores plus libres qui s'organiseraient en quelque sorte en réseau autour d'elles. Nous allons nous employer à illustrer ce phénomène.

\subsection{Métaphores non figées et champs métaphoriques}

\subsubsection{Les catastrophes naturelles : tempêtes en mer, séismes}

Nous avons eu l'occasion de mentionner ce registre dans nos exemples de métaphores lexicalisées. Une des aires couvertes par l'expérience humaine, celle des caprices du temps, des désastres naturels, explique que, logiquement, une crise sur les marchés financiers est décrite en termes de tempête dévastatrice ( « currency turmoil »). La récente crise asiatique (fin 1997-début 1998) dont les conséquences se font encore sentir, a fourni maintes occasions aux commentateurs et analystes de se livrer à des variations sur ce thème donné :

Asia's financial storm grew to hurricane force (The Economist, June 6, 1998, " Hong Kong's Economy : gloomy »)

As the full force of the financial typhoon hits the region's economies, ... ( Business Week, June 1, 1998, « Asia : the Global Impact »)

A wave of currency depreciation has swept through East Asia ... (The Economist, November 1, 1997, « The ABC of a Currency Board »)

Analysts say Asian cash now comprises a big chunk of the \$ billion pouring into U.S large-cap and index mutual funds each week. Since mid-March, inflows into European equities through U.S-based mutual funds have nearly tripled, to over $\$ 300$ million a week... The torrent also has buoyed the dollar and kept U.S interest rates lower than they might otherwise have been. ( Business Week, June 1, 1998, « Flight to Safety »)

Contre vents et marées, il ne reste plus qu'à se protéger ou trouver un havre rassurant :

Since a financial crisis forced Thailand to allow its currency to float on July 2 (and then sink), some of its neighbours have had to strap their lifebelts on theirs. The Philippine peso is finding it hardest to keep its head above water. ( The Economist, July 12, 1997, « Asian Currency Jitters ») 
The collapse of Asia's currencies unleashed a flood of foreign investment seeking the safety of U.S markets. (Business Week, June 29, 1998, "Currencies »)

Investors have sought a safe haven for their funds. (The Financial Times, June 8, 1998, « Survey : Foreign Exchange »)

Quant aux séismes, ils donnent aussi la possibilité de fournir au lecteur ou à l'interlocuteur un élément de référence :

It seems that for emerging economies there's no shelter from the aftershocks of East Asia's financial crisis. Now the tremors have reached South Africa, shaking its economy... (Time, July 13, 1998, " The Speculators' Latest Target »)

Nous ne multiplierons pas les exemples pour ne pas alourdir cette étude, mais nous avons souhaité illustrer la marque individuelle que chaque auteur peut imprimer à la présentation qu'il fait de la réalité par le biais de la métaphore, tout en restant dans un même registre. Il apparaît donc possible d'avancer que les métaphores lexicalisées font partie de la langue en tant que système alors que les métaphores non lexicalisées font partie de la parole puisqu'elles sont des manifestations effectives du langage, lieu des variations individuelles (Sarfati $1997: 9$ )

Quant au problème de savoir si nous sommes en présence de métaphores d'ornement ou si ces métaphores sont porteuses d'un message, nous dirons qu'il serait erroné de renvoyer dos à dos ces deux aspects : nous y reviendrons dans notre dernière partie sur l'analyse du discours métaphorique, mais nous pouvons d'ores et déjà dire que, s'il y a effet de style pour certains, il n'exclut pas (et trouve peut-être ici sa justification) un souci de rendre moins opaques les mécanismes assez complexes des systèmes monétaires dans le contexte de la mondialisation et d'illustrer leurs conséquences en chaîne par le recours à ce que chacun connaît, en décrivant la crise en termes de vague déferlante ou de bateau en perdition.

\subsubsection{Registre militaire, du combat, de la lutte...}

Le registre militaire s'applique naturellement, lui aussi, au domaine des monnaies dans la mesure où, pour faire face à des difficultés, à une crise monétaire, il faut mettre au point une stratégie pour se défendre ou attaquer, déployer des forces pour se battre :

Currency boards can help countries parry attacks on their currencies. (The Economist, Nov 1, 1997, "The ABC of a Currency Board »)

Critics insisted that the Reserve Bank's apparent dithering over the assault on the rand was part of the problem. (Time, July 13, 1998, "The Speculators' Latest Target »)

Because Japan is a major player in world markets, a massive run on yen could destabilize the global financial system. (Business Week, June 29, 1998, « A Day at the Front in the Currency Wars »)

\subsubsection{Registre de l'envol, de l'escalade ou de la chute libre}

Les fluctuations des monnaies peuvent être très fortes, dans le sens de la hausse comme dans celui de la baisse, et se traduisent soit par des métaphores nautiques, comme nous l'avons vu, où une forte baisse est assimilée à une noyade ou un naufrage, soit par des métaphores empruntées au registre de l'escalade (" the ascent of the dollar »), de l'envol 
("the soaring dollar ») ou du parachutisme, où la chute libre ne peut durer qu'un laps de temps si on veut éviter la catastrophe :

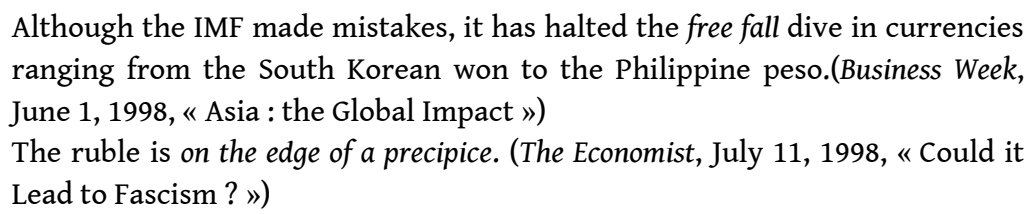

\subsubsection{Registre de la santé, personnification} les spéculateurs comme pour les autorités, et les cours sur le marché des changes reflètent les forces et les faiblesses des monnaies respectives : c'est alors que « a strong dollar " peut devenir "the robust dollar ", "the muscular greenback", face à d'autres monnaies moins heureuses qui sont, elles aussi, décrites de manière plus métaphorique : « the fragile yen » ou « the shaky ruble ».

We don't believe our ringgit fell weak, soft-kneed and collapsed, [Malaysia's prime minister Mahathir Mohamad] opined. (The Economist, November 29, 1997, « Asia »)

By now, according to the original prognoses, the patients should be out of intensive care. Weak but convalescent, they should at least have a glimpse of recovery. Yet Asia's three big recipients of IMF-administered transfusions Thailand, Indonesia and South Korea- are still in a critical condition. (The Economist, July 11, 1998, «Still Sick and Gloomy, now Rebellious »)

Quant à l'impact que peuvent avoir les difficultés rencontrées par une monnaie sur l'économie mondiale, il est décrit en termes de maladie contagieuse :

Why is turbulence in the currency markets infectious? And what decides whether any given currency is likely to succumb ?... Thus, ... the emergingmarket contagion of 1995 had mainly 'rational' causes (The Economist, August 31, 1996, « Are Crashes Catching?»)

\subsubsection{Registre de la construction d'un édifice}

Empêcher une monnaie de s'effondrer revient à la consolider en la renforçant (« to prop up the yen »), comme on le fait pour une construction:

...the next step is to push Japan for the reforms that could give the yen a solid foundation. (Business Week, June 29, 1998, « Asia »)

...this summer, the crumbling ruble has the alarm bells ringing louder than ever. (Time, July 13, 1998, « Time to panic?»)

Robert Rubin, America's Treasury secretary, wants to 'modernise the architecture of the international financial markets'. (The Economist, April 11, 1998, « The Perils of Global Capital »)

33

Les exemples cités permettent de conclure à l'existence de liens entre les divers domaines d'emprunt : non seulement les métaphores non-lexicalisées sont reliées aux métaphoressouches dont elles découlent, mais elles sont également interconnectées. En effet, nous pouvons nous rendre compte de ces liens en examinant la logique sous-jacente. Les registres d'emprunt que sont les éléments naturels déchainés, la guerre et la maladie 
partagent les sèmes de la lutte et de la crise : on se bat contre la tempête comme on se bat contre un rival et contre la maladie. Les domaines de la construction, de la marine, de l'escalade et de la santé ont en commun le sème du risque d'échec, traduit respectivement par l'effondrement, le naufrage, la chute et la mort. Or, pour éviter l'échec et gagner le combat, il faut précisément rassembler des forces, autre point commun qui consolide la trame tissée entre ces différentes métaphores. C'est sans doute ainsi que peut s'expliquer l'existence de constantes dans les métaphores rencontrées dans l'ensemble des sources que nous avons consultées, quel que soit le degré de technicité de ces documents.

Il s'agit maintenant, en gardant à l'esprit les registres métaphoriques recensés dans le discours sur les monnaies, de mettre en parallèle les métaphores utilisées pour parler plus particulièrement de l'euro afin d'analyser les caractéristiques du discours propre à la monnaie unique et d'essayer d'en tirer un enseignement.

\section{L'euro}

\subsection{Remarques préliminaires}

Avant même d'aborder le discours métaphorique, il nous a semblé souhaitable de rappeler que l'euro est différent des autres monnaies à plusieurs titres. Tout d'abord, s'il succède à l'écu, qui était déjà particulier en ce qu'il pouvait être assimilé à une monnaie virtuelle, bien que calculé à partir d'un panier de monnaies existantes dont il était la moyenne, l'euro sera différent : il aura une existence propre et il sera matérialisé par des pièces et des billets qui remplaceront les monnaies des pays qui l'auront adopté. Ensuite, contrairement aux monnaies actuelles, considérées comme symboles de l'identité nationale aux côtés de l'hymne, l'armée ou le drapeau, l'euro ne pourra pas être identifié à une seule nation. Enfin, même si l'euro n'est pas la première tentative de création d'une monnaie unique, puisqu'il y a eu dans l'histoire plusieurs expériences dans ce sens, il faut dire que le seul exemple actuel, à savoir le dollar, n'est pas le résultat d'une démarche comparable. En effet, comme le faisait très justement remarquer Christine Ockrent (1998) lors d'un récent colloque sur l'euro à l'Assemblée nationale, à la différence du dollar, né dans le sang de la guerre d'indépendance, l'euro est né de la paix et du désir de la préserver.

\subsection{Unités néologiques et phraséologismes à connotation métaphorique (Annexe 1)}

La démarche que nous avons adoptée consiste à nous attacher aux unités courtes à valeur nominale (mots simples ou composés) avant de poursuivre un itinéraire qui passe par les phraséologismes plus longs (incluant adjectifs et verbes en collocation) pour arriver enfin au discours métaphorique et à son analyse. Autrement dit, nous nous sommes d'abord placée d'un point de vue lexicologique (métaphore-mot) pour ensuite envisager le point de vue sémantique et stylistique (métaphore-discours).

La liste que nous avons établie pour notre étude des néologismes autour de l'euro (Resche 1998) nous a donné l'occasion d'ouvrir un certain nombre de pistes que l'examen des phraséologismes en Annexe 1 semble confirmer et préciser. Les termes transcrits en italiques rappellent simplement que certains registres relevés à propos des monnaies en 
général valent aussi pour l'euro. Il importe maintenant de relever les nuances et les différences qui ressortent de l'examen de cette liste au plan métaphorique.

\subsubsection{L'euro traité en être humain : naissance, santé physique et morale} de la zone euro, c'est-à-dire la zone dans laquelle l'euro sera utilisé, et les phraséologismes relevés renvoient alors à la notion de club fermé (" euro membership ", " member of the euro ", " to join the euro ", « to be admitted in the euro »). Ce " club euro" ( euro club») demande de la part de ses adhérents un certain engagement («tie to the euro », « commitment to the euro »).

\subsubsection{L'euro : une religion}

L'engagement requis pour être accepté dans le « cercle de l'euro » est tellement fort qu'il peut prendre une autre dimension en se transformant en engagement religieux. C'est ainsi qu'un terme tel que "to believe in the euro", mis en parallèle avec d'autres expressions comme " to convert to the euro " ou " to bow to the euro ", " to be worthy of the euro » et «the advent of the euro ", nous conduit à penser que l'euro est ici érigé en 
religion et que son arrivée est attendue comme celle d'un messie. La ferveur pourrait même aller jusqu'au fanatisme (« euro zeal », « euro zealot »)!

\subsubsection{L'euro : un combat} que les positions sont heurtées entre les eurosceptiques, les europhiles et les europhobes. Chaque camp ("anti-euro camp », "defenders of the euro ») met en place une stratégie (" euro strategy ») et est prêt à se battre (" anti-euro warriors ", " euro campaign », " the casualties of the euro $»)$.

\subsubsection{L'euro et les moyens de transport}

L'aventure de l'euro devient une expédition, et les métaphores passent alors par le registre des moyens de transport. L'allusion à un lancement de fusée, amorcée par «to launch ", est confirmée par d'autres termes, en l'occurrence " euro countdown», « to get off the ground » et «euro boosters». L'autre métaphore est celle du train, avec « eurotrain » et le verbe « to derail », qui donne à l'éventualité d'un échec de l'euro la dimension d'une catastrophe dont chaque lecteur peut aisément mesurer l'ampleur.

Les registres métaphoriques de l'euro passés en revue jusqu'alors sont simplement le résultat de l'examen des néologismes et phraséologismes recensés dans le cadre d'une autre étude (Resche 1998). Il convient de franchir le pas vers des énoncés plus longs afin d'affiner l'analyse, de vérifier si ces registres sont confirmés et de les compléter le cas échéant.

Pour Lakoff et Johnson (1980), il existerait des méga-métaphores, ce qu'ils appellent « systematic metaphors", qu'ils définissent ainsi : "metaphors relating to the same topic [that] can be grouped together in a mega-metaphor on a semantic basis ». Nous trouverons peut-être réponse à cette question en élargissant notre examen au contexte de la phrase ou de plusieurs phrases dans le cas de métaphores filées.

\section{3. Énoncés métaphoriques et «méga-métaphores » (Annexe 2)}

Puisqu'il est vrai que «l'effet de sens résulte d'une certaine action que les mots exercent les uns sur les autres dans la phrase» (Ricœur 1975: 99), ce sont bien les énoncés métaphoriques qu'il faut observer car c'est la coexistence de termes non métaphoriques et de termes pris dans un sens inhabituel pour le contexte qui fait ressortir et comprendre les métaphores. Si nous avons pu déceler les connotations métaphoriques dans des néologismes ou dans des phraséologismes relativement courts, c'est simplement parce que nous connaissions le contexte sous-jacent de l'euro ou parce que « euro » se trouvait en composition dans ces termes.

D'autre part, la connaissance du contexte est primordiale pour mieux appréhender le sens voulu par le lecteur ou l'auteur. Considérons, en effet, l'expression « to emasculate the euro "; hors contexte, on pensera spontanément que cette expression fait allusion à un acte de sabotage voulu par des opposants à l'euro qui cherchent à nuire au projet. Mais, si l'on prend connaissance de l'énoncé, on s'aperçoit que l'expression qui, certes reste forte, s'applique à une attitude française jugée trop laxiste par les Allemands : il s'agit d'une interprétation moins stricte des critères de convergence soupçonnée d'affaiblir la future 
monnaie unique. Or on sait que l'intention de la France n'est pas de mettre l'euro en péril et qu'il n'y a pas eu de malveillance délibérée : «German officials insist they will resist any French measures that might emasculate the euro » (Newsweek, January 13, 1997, « Europenny Opera »).

Nous avons évidemment dû opérer une sélection des illustrations, tant les exemples étaient nombreux, et nous n'avons conservé que les métaphores communes aux divers niveaux de discours: s'adressant à des lectorats différents, elles s'éclairent ainsi mutuellement. Afin de ne pas allonger démesurément le corps de cette étude, nous avons choisi de citer ces contextes en Annexe 2 et nous les avons numérotés de sorte que nous nous contenterons d'indiquer les chiffres entre parenthèses lorsque nous $\mathrm{y}$ ferons allusion.

D'entrée, nous pouvons dire que les domaines d'emprunt s'avèrent confirmer les indications fournies par les unités plus courtes, et ceci est intéressant car nous sommes en droit de penser qu'une analyse détaillée des néologismes à base nominale, complétée par un examen des phraséologismes, constitue une source de renseignements assez révélateurs quant au discours sous-jacent. La différence réside dans une déclinaison plus large des métaphores dans les énoncés examinés. Ainsi, la métaphore de l'être humain, que nous avions également signalée pour les autres monnaies, est beaucoup plus développée ici, puisque ce sont toutes les étapes d'une vie qui sont envisagées depuis la gestation (5). Tour à tour, sont mentionnés la naissance (1 à 5), l'enfance (4), les fiançailles (7) et le mariage (7) qui supposent l'amour (6), et inévitablement les étapes moins agréables telles que la maladie (8) et bien sûr la mort (9). Le chemin vers l'euro est décrit en termes de bosses et nids de poules (20-21), ou transposé en course d'obstacles (24), ce qui permet d'emprunter au registre de la compétition sportive (22-23). Quant au domaine des transports, là aussi les métaphores-phrases sont plus variées en ce sens qu'elles vont de la bicyclette (16) à l'avion (19), à la fusée précédemment mentionnée, en passant beaucoup plus longuement par le train (13 à 18) que ne le faisaient les métaphores-mots. Enfin, il est assez impressionnant de constater comment l'euro est sanctifié, si l'on en juge par l'enthousiasme du premier ministre portugais qui est allé jusqu'à comparer l'euro à Saint Pierre sur qui l'Église a été bâtie, en déclarant : « the single currency will be the rock for Europe » (The Economist, December 23, 1995, « Single-currency Minded »).

51 Nous aimerions nous arrêter un instant sur la métaphore du mariage, que nous n'avions pas mentionnée dans notre analyse des métaphores-mots, car il nous semblait que, même si nous avions relevé "euro honeymoon", cela n'était pas suffisant pour conclure à l'existence d'un champ métaphorique. Il se trouve que les énoncés plus longs nous ont permis de confirmer cette hypothèse. Jusqu'alors, en langue économique, cette métaphore était naturellement réservée aux fusions d'entreprises, mais on conçoit son extension à la monnaie unique qui résulte précisément de la fusion de plusieurs monnaies en une seule.

À un autre titre, ce registre est intéressant, car il nous permet de voir comment fonctionne la logique des locuteurs ou auteurs, dans la mesure où, dès lors qu'un champ métaphorique est ouvert, il peut se concevoir au positif comme au négatif. C'est ainsi que la métaphore du mariage aura pour négatif celle du divorce et que les termes de ce registre dans la langue courante pourront s'utiliser dans le discours métaphorique (« the break-up of the euro »). De la même façon, la métaphore de la construction solide pourra être reliée à une métaphore contraire suggérant le manque de solidité, l'effondrement de l'édifice, et nous concevrons par expérience qu'elle appelle l'idée du cataclysme. Nous 
avions déjà évoqué la notion de liens entre métaphores de domaines différents, de réseau formé par des sèmes communs à plusieurs registres, et l'on sait qu'il suffit d'un seul sème commun pour créer la métaphore et pour relier entre elles plusieurs métaphores. Ce phénomène se vérifie ici, puisque, par exemple, la métaphore de l'euro considéré comme un club évolue en métaphore du mariage (12) par le simple fait que ces deux registres ont en commun le sème de l'association de personnes (plusieurs pour le club, deux dans notre conception du mariage) ayant envie de partager quelque chose. Dans ce cas précis, il y a aussi analogie entre la fidélité au club (monnaie stable, discipline économique) et fidélité à l'engagement pris, stabilité et loyauté dans le mariage.

Enfin, nous avons voulu illustrer en Annexe 3 un passage plus long qui est un morceau de bravoure au plan stylistique parce qu'il constitue un exemple parfait du processus de remétaphorisation que nous avons décrit plus haut et en même temps une mise en pratique du phénomène d'entrecroisement des métaphores. Il s'agit de la transcription de «l'effet Maastricht » en termes d'ouragan, en conformité avec les exemples donnés au sujet des autres monnaies, où l'Union européenne est vue comme un bateau aux prises avec une terrible tempête qui met sa structure à dure épreuve et qui risque de faire beaucoup de victimes. Ce sont donc trois domaines métaphoriques qui s'entrelacent ici, celui de la tempête, d'un bâtiment ou d'une construction et celui de la santé, de la personnification. Nous avons mis en italiques les différents termes de ces métaphores pour montrer comment une métaphore considérée comme lexicalisée, banalisée, peut renaître de ses cendres parce que, sur le canevas de base, l'auteur peut broder à loisir; un point en appelle un autre et la tapisserie s'enrichit ainsi.

De tous ces exemples, il ressort que le cas de l'euro met encore davantage en relief les liens logiques qui unissent les métaphores les plus fréquentes, qui sont celles qui nous intéressent dans la présente étude. Et si nous avons pu remarquer des registres communs avec le discours des autres monnaies, nous avons aussi constaté la plus grande variété de la gamme des métaphores concernant l'euro, comme si, du fait de cette expérience encore inédite, il était besoin d'en appeler davantage à l'imagination et de l'asseoir sur une expérience déjà connue.

\subsection{Le discours métaphorique}

Un récent article du Financial Times (June 5, 1998, «Foreign Exchange Survey : Strong Line Shifts into Neutral») soulignait, s'il en était encore besoin, l'impact de la langue sur l'événement, à l'occasion des propos tenus pas les autorités monétaires américaines sur le dollar :

In 1995, Mr Rubin and his colleagues had a verbal policy for the dollar that was simple and declarative : «We favour a strong dollar »... But a year ago, the formula changed... To the now familiar mantra, he added a coda : « and we have had a strong dollar for some time now. »

Et le Financial Times d'ajouter : «What do these tortuous gymnastics amount to ? Has the US stopped talking up the dollar? » Cette dernière expression, soulignée par nos soins, résume à elle seule l'impact du discours sur les réactions du marché et nous conduit tout naturellement à nous poser la question du rôle des métaphores étudiées et leur effet sur le public en général. Comme nous le rappelle Jean-Noël Jeanneney (1998:1) dans un éditorial :

L'Histoire nous l'enseigne : jamais au long des siècles, la monnaie n'a été un élément neutre et transparent, jamais un instrument purement technique. Mais toujours un 
révélateur des mentalités et un acteur essentiel dans le jeu des passions collectives et de la raison en marche. sera par essence: le résultat d'une fusion entre diverses monnaies, mais une monnaie nouvelle, baptisée d'un nom inédit avant même sa naissance réelle. Ce fut une entreprise de longue haleine, semée de difficultés, confrontée à l'incrédulité de certains, et dont l'aboutissement sera une sorte de couronnement. C'est aussi parce qu'il n'est pas encore réalité que l'euro est investi de ce caractère quasi religieux : on ne peut qu'y croire ou non, qu'avoir confiance, foi en ce projet ! Seuls les détails techniques, théoriques, sont à la disposition du public, mais l'expérience, la pratique, font défaut et c'est précisément là qu'intervient le discours métaphorique. D'une part, il vise à exprimer les craintes, la désapprobation ou, au contraire, les espoirs, la confiance, voire l'enthousiasme, c'est-àdire qu'il est là pour révéler, refléter les mentalités; d'autre part, il sert à essayer de convaincre, soit d'abandonner le projet, en insistant sur ses côtés négatifs, soit de persévérer en expliquant et en se voulant résolument rassurant, et dans ce sens, il forme les mentalités. Selon les cas, le discours métaphorique est donc rhétorique, persuasif, et/ ou didactique. À cet égard, le caractère iconique des métaphores, qui font voir les ressemblances en s'appuyant sur le vécu du lecteur, est poussé encore plus loin : en effet, des dessins, illustrations littérales des propos tenus, accompagnent de nombreux articles, matérialisant ainsi les images mentales suggérées par le discours métaphorique; ils mériteraient, à eux seuls, une étude parallèle.

La question qui reste entière est celle de l'adéquation entre l'intention de l'auteur de la métaphore et l'effet produit sur le destinataire du message. S'agissant du niveau illocutoire, d'abord, c'est-à-dire «du but que l'énonciateur veut atteindre » (Larreya \& Watbled 1994: 69), nous devons garder à l'esprit la multiplicité des énonciateurs impliqués dans notre étude. Selon les cas, il peut s'agir d'un journaliste-chroniqueur ou d'un éditorialiste qui viseront à préserver leur lectorat et donc à véhiculer le discours attendu, ou bien d'un spécialiste d'économie qui voudra persuader que son analyse est pertinente, ou encore d'un technicien désireux d'expliquer les mécanismes économiques, ou enfin d'un homme politique cherchant à justifier sa position. Le discours métaphorique, s'il passe par les mêmes registres d'emprunt, résulte donc de motivations différentes. Il importe également de se demander si l'auteur poursuit réellement un but en ayant recours aux métaphores, s'il est conscient de ce procédé qu'il utilise ou s'il est simplement soucieux de jouer avec les mots par amour des belles phrases. Enfin, nous ne pouvons écarter un effet de contagion, de mode, qui fait que l'on repasse par des formules lues ici ou là et des tics de langage. Le niveau perlocutoire (Larreya \& Watbled 1994: 69) est encore plus difficile à appréhender. En d'autres termes, quand bien même l'auteur est conscient du discours 
métaphorique qu'il tient, comment peut-il être certain que son message sera reçu et compris comme il l'a voulu? Assurément, les opinions du lecteur peuvent varier au gré des derniers développements de l'actualité, et une même métaphore pourra être perçue de manière négative ou positive. Par exemple, pour qui est persuadé que la monnaie unique est un atout pour l'Europe, il est évident que la métaphore du mariage trouvera un écho positif; au contraire, l'eurosceptique ou l'europhobe y verront un côté négatif, considérant que mariage peut signifier perte de liberté, d'autonomie et d'identité. Il suffit parfois d'un grain de sable dans l'engrenage du processus de la monnaie unique pour que celui qui était bien disposé à l'égard du projet se mette à douter et change de camp. L'accueil qu'il réservera à cette métaphore en sera affecté.

61 Les dates des énoncés sont également à prendre en compte car elles peuvent nous éclairer sur l'évolution des idées. Par exemple, les métaphores des cataclysmes et des tempêtes se font l'expression de moments de découragement, de pessimisme, de tensions, où les difficultés rencontrées font envisager l'échec du projet: ainsi, l'attente de résultats très incertains lors d'élections importantes pour l'Union Monétaire se lit dans les métaphores. De la même manière, maintenant que des caps difficiles ont été franchis et que l'on sait que l'euro se fera à onze dans un premier temps, les métaphores évoluent : elles se font plus sereines quant à l'échéance qui approche, et font davantage allusion au problème de savoir si le train pourra supporter des wagons supplémentaires, ou si le club doit ouvrir sa porte à d'autres membres, dans l'optique d'une ouverture à l'Est. Une étude diachronique du discours métaphorique de l'euro pourrait donc nous apprendre bien des choses quant à l'évolution des mentalités, et un élargissement de cette étude à d'autres langues nous permettrait certainement de déterminer si tous les Européens tiennent le même discours métaphorique et d'ajouter une dimension culturelle en relevant les différentes expressions d'une même réalité selon les langues et la sensibilité des peuples.

\section{Conclusion}

Pour terminer, nous souhaiterions rappeler un certain nombre de points établis au cours de cette étude. Tout d'abord, l'on se souviendra que la langue spécialisée n'est pas une langue à part et qu'elle prend sa source dans la langue commune: de là découle notre position selon laquelle, de la même manière que les mots isolés ne sauraient servir à appréhender une langue, la métaphore-mot ne peut suffire à comprendre le sens métaphorique d'un discours. Si l'on ne doit pas ignorer le mot, il faut cependant élargir l'étude à l'énoncé métaphorique au niveau de la phrase au moins.

Ensuite, en langue spécialisée, la métaphore ne peut se concevoir dans une fonction purement ornementale; elle est, en revanche, motivée par un souci d'expliquer ou de convaincre ou les deux à la fois. Nous avons souligné ses fonctions rhétorique, lexicale parfois, aussi bien qu'iconique et didactique. Il ne faut pas non plus négliger l'aspect euphémique qui permet de transformer une tempête en brise et de minimiser ainsi l'impact d'une crise, donc, dans ce cas, de rassurer pour influencer l'opinion.

Enfin, nous insisterons sur la spécificité de l'euro, cette prochaine monnaie unique dont on parle tant et qui n'a pas encore vraiment d'existence, ou plutôt qui est déjà présente avant même d'être née tant elle fait couler d'encre! Cette spécificité fait, en quelque sorte, l'intérêt de cette étude, en ce qu'elle permet d'apprécier la façon dont le discours métaphorique, privilégié ici puisque l'euro ne vit encore que dans l'imaginaire collectif, peut être à la fois miroir des pensées et moteur des opinions. Les mois à venir devraient 
s'avérer riches en enseignement, qu'il s'agisse de la période de transition ou des premiers pas de «l'enfant». Gageons que la métaphore de la construction sera déclinée différemment dans la perspective de l'élargissement à d'autres pays. Nous pouvons également nous attendre à voir les métaphores couramment utilisées pour les autres monnaies s'appliquer à l'euro une fois qu'il sera soumis aux caprices du marché, qu'il fera l'objet d'échanges et de spéculation, qu'il rivalisera peut-être avec le dollar jusqu'à devenir l'autre devise de réserve. Quoi qu'il en soit, il apparaît que le discours métaphorique qui a fait l'objet de cette étude, loin d'être banalisé, révèle bien plus que de simples effets de style: c'est un véritable baromètre des mouvements de pensée et des réactions d'une époque donnée.

\section{BIBLIOGRAPHIE}

Aristote. 1969. Poétique, traduction française de J.Hardy. Paris : Les Belles Lettres.

Benvéniste, Émile. 1966. Problèmes de linguistique générale, Tome 1. Paris : Gallimard.

Cicéron. 1930. De l'orateur, traduction de E. Courbaud. Paris : Les Belles Lettres.

Corbisier, Agnès. 1994. « Metaphor not as a stylistic but as a cognitive device : English-language information technology manuals as a test-case ». ASp 4, 43-59.

Jeanneney, Jean-Noël. 1998. «L'euro et après ? ». Europartenaires : la lettre de l'association 8, 1.

Lakoff, George et Mark Johnson. 1980. Metaphors We Live By. Chicago, IL : University of Chicago.

Le Guern, M. Sémantique de la métaphore et de la métonymie, Paris : Larousse, 1978.

Larreya, Paul et Jean-Philippe Watbled. 1994. Linguistique générale et langue anglaise. Paris : Nathan.

Ockrent, Christine. 1998. Communication faite à l'occasion d'une table ronde dans le cadre du «Colloque l'Euro : plus rien comme avant ? Les dimensions politiques et culturelles d'un choix historique ", organisé conjointement par Europartenaires, Témoin et la Fondation Friedrich Ebert, tenu à l'Assemblée Nationale le 2 juillet 1998.

Resche, Catherine. 1998. « De l'Europe à l'euro, ou 'l'industrieuse activité de la langue' : recensement et analyse des néologismes en anglais et en français entre 1991 et 1998 ». ASp 19-22, 185-208.

Ricœur, Paul. 1975. La métaphore vive. Paris : Éditions du Seuil.

Sarfati, Georges-Elia. 1997. Éléments d'analyse du discours. Paris : Nathan Université.

Swales, John M. 1990. Genre Analysis. Cambridge : Cambridge University Press.

Tournier, Jean. 1985. Introduction descriptive à la lexicogénétique de l'anglais contemporain. Paris, Genève : Champion-Slatkine.

Tournier, Jean. 1993. Précis de lexicologie anglaise. Paris : Nathan Université.

Bibliographie complémentaire

Baylon, Christian et Xavier Mignot. 1995. Sémantique du langage. Paris : Nathan. 
Cotte, Pierre et al. 1993. Les cahiers de la grammaire anglaise en France. Paris : Hachette Supérieur.

Partington, Alan. 1995. « A Corpus-based investigation into the use of metaphor in British business journalism ». ASp 7-10, 25-39.

Sweetser, Eve. 1991. From Etymology to Pragmatics. Metaphorical and Cultural Aspects of Semantic Structure, Cambridge Studies in Linguistics 54. Cambridge : Cambridge University Press.

\section{ANNEXES}

Annexe 1 : Rappel des néologismes (Resche 1998)

\begin{tabular}{|l|l|}
\hline euro adoption & \\
euro big bang & \\
euro boosters & euro-aspirants \\
euro campaign & euro-capabilities \\
euro club & euro-conversion \\
euro countdown & euro-day \\
euro era & euro-launch \\
euro honeymoon & euro-stakes \\
euro membership & euro-train \\
euro midwife & euro-worthiness \\
euro participants & euro-worthy \\
euro revolution & euro-zeal \\
euro start & euro-zealot \\
euro strategy & \\
euro finish-line & \\
\hline anti-euro camp & \\
anti-euro warriors & \\
\hline
\end{tabular}

\section{Rappel des phraséologismes}




\begin{tabular}{|l|l|}
\hline $\begin{array}{l}\text { advent of the euro } \\
\text { age of the euro }\end{array}$ & \\
break-up of the euro & commitment to the euro \\
casualties of the euro & path to the euro \\
defenders of the euro & run up to the euro \\
fan of the euro & tie to the euro \\
viability of the euro & \\
\hline
\end{tabular}

\section{L'euro et ses adjectifs}

\begin{tabular}{|l|l|}
\hline beloved & \\
faltering & soggy \\
flabby & solid \\
flaccid & stable \\
fragile & strong \\
mushy & tough \\
robust & weak \\
rock-hard & weakish \\
shaky & wobbly \\
\hline
\end{tabular}

\section{L'euro et ses verbes}




\begin{tabular}{|c|c|}
\hline $\begin{array}{l}\text { euro = sujet du verbe } \\
\text { to be born } \\
\text { to be due to arrive } \\
\text { to come into being } \\
\text { to get off the ground }\end{array}$ & $\begin{array}{l}\text { euro = complément du verbe } \\
\text { to adopt the euro } \\
\text { to be admitted in the euro } \\
\text { to believe in the euro } \\
\text { to bow to the euro } \\
\text { to challenge the euro } \\
\text { to convert to the euro } \\
\text { to debauch the euro } \\
\text { to deliver the euro } \\
\text { to derail the euro } \\
\text { to embrace the euro } \\
\text { to hit the euro } \\
\text { to inaugurate the euro } \\
\text { to kill the euro } \\
\text { to join the euro } \\
\text { to launch the euro } \\
\text { to like the euro } \\
\text { to love the euro } \\
\text { to mistrust the euro } \\
\text { to oppose the euro } \\
\text { to qualify for the euro } \\
\text { to undermine the euro }\end{array}$ \\
\hline
\end{tabular}

\section{Annexe 2 : Les énoncés métaphoriques à propos de l'euro}

Personnification : vie (gestation, avortement ?, naissance, amour, fiançailles, mariage, maladie, mort)

The European Union is understandably obsessed by worries about the birth of its single currency. But Europeans should remember that their family grows by adoption as well as reproduction. (The Economist, June 14, 1997, « Widen Europe »)

Plenty of people doubt if Europe's single currency will be born on time -or at all. (The Economist, April 5, 1997, « European Monetary Institute : Preparing to Die »)

Creating a single European currency is a good idea, but it must be born strong and viable. ( Business Week, June 24, 1996, « Monetary Union : By Hook or by Crook »)

Will Europe's planned single currency start on the due date of January 1, 1999 ? The answer is still probably yes - but it could have a blighted infancy... If it is a strong currency, buttressed by tight monetary policies, then its early years could well be painful ones for Europe's weaker economies... No rule says Mr Kohl cannot lose an election, and if 
Mr Schroeder were to emerge victorious, that would cloud - and could conceivably abort - the single currency's birth. (The Economist, June 7, 1997, « Kicking and Screaming into $1999 »)$

The baby's birth, at least, is certain. After a long gestation, the markets have accepted EMU. (Newsweek, May 4, 1998, Special Report. «The Era of the Euro Arrives »)

Instead of blossoming into a torrid affair, Europe's flirtation with the single currency has been caught in a sudden freeze... But if there is a second-half rebound this year, as the Commission now predicts, the clouds of gloom will lift, and the single currency could enjoy a summer romance. (Time, 1966, « Money Unity in Name Only »)

If Europe cannot forge ahead with plans for political union, what's the point of a common currency? Regardless of this fact, prenuptial agreements to a communal marriage of the mark, franc, lira, etc., have already been signed, with the wedding date scheduled for 1999. ( Business Week, September 25, 1995, « Common Money -or Funny Money?»)

It is an exercise in healing, the latest and most important bandage placed on the continent's old wounds. (Newsweek, May 4, 1998, «The Era of the Euro Arrives »)

Kohl is probably right to fear that a delay would spell the death of the euro. (Business Week, March 31, 1997, «Put the EMU on the Endangered List »)

\section{Club}

...it's a club that offers companionship, broadly like-minded partners and a vision -however blurred- of a future with friends around. (Time, March 14, 1994, « Now there are $15 »)$

...enlargement will also involve less palatable changes, simply because $a$ club of 25 or 27 cannot operate with rules designed for a club of six or even one of 1...Indeed, the larger a club becomes, the smaller the number of activities in which all of its members are likely to want to take part. (The Economist, March 30, 1996, « Europe Tries Again »)

You can't join a club by observing rules that are less stringent than those that apply in the club... The criteria have to apply equally to all. If you want a stable currency, it won't do if one country respects the criteria and another acts in a different way. For better or worse, this is a marriage without a dissolution. (Financial Times, July 7, 1997, « Defender of a Decimal Point »)

\section{Transports (rail, route, air)}

...And then there is the question of where to draw the line : Italy, yes -but Greece, Portugal and Spain? First class is full; perhaps there will be some coach seats for the underachievers. (Business Week, September 25, 1995, «Common Money -or Funny Money ?)

It would be better to delay the train than face derailment later... To recall the Tietmeyer theorem : « it is better to delay departure than to derail outside the station». Behind this metaphor lurks an incontrovertible piece of economic logic. Unless all the cars move reliably at the same speed and in the same direction, the couplings won't hold and the rails will buckle. (Time, April 1, 1996, « Caught in the Maastricht Grinder »)

With the EU summit in May set to formalize the euro decision, they know that the train is pulling out of the station. (Newsweek, February 2, 1998, « The Global Economy. Once More Around the Track») 
The Europeans have a bicycle theory of integration -it's got to go forward or it stops. ( Newsweek, January 13, 1997, « Europe's New Nutty Money »)

« All aboard the Franco-German-Benelux Express. Destination : Little Europe, perhaps as early as 1997 »...The Bundestag's major parties are terrified by what the world would think if Germany rejected Europe ? So the train, maybe, will run on time. Yet, the consequences of a reduced passenger load would be serious. (Time, September 28, 1992, « And Now, Vox Populi »)

As Mr Baldwin puts it, « unpleasantness is unavoidable if second-class ticket holders can vote on what first-class passengers will have for dinner ». (The Economist, April 16, 1994, «Poor Men at the Gate »)

...revving up for the euro's maiden flight... (Conjoncture, March 1998, no. 3, Paribas)

\section{Chemin difficile}

But there are many bumps ahead that could hurt the euro's momentum, or even derail it. ( Business Week, April 27, 1998, « Special Report. The Euro »)

« It's going to be a very bumpy road to get to 1999 », says Neil MacKinnon, chief economist for Citibank in London. Some of the biggest potholes are Maastricht's criteria for joining the single currency. (Time, July 3, 1997, « Recognizing Reality »)

\section{Compétition sportive}

So there is more than just the euro to try Europe's soul (and stomach) in the next several years. It is " deepening " and « widening » all at once - adding muscle and speed while trying to gain weight. Boxers preparing for the heavyweight championship have occasionally succeeded at this tortuous game. But the EU, a bunch of 15 (and soon 20) nations, is no Muhammad Ali... If truth must be told, none of the others (with the exception of tiny Luxembourg) will have qualified either. Each and all have merely made a desperate dash toward the finish line, especially the magic $3 \%$ limit on the deficit as a fraction of the gross domestic product. They have done so not by sweating off fat but by shooting up with steroids... (Time, January 19, 1998, Special Report. « Countdown to E-Day »)

Curiously, most of the talk has focused on the rather arbitrary budgetary and « convergence » criteria involved in getting the euro across the starting line. Far less attention has been paid to the more fundamental changes needed to reach the finish line...( Newsweek, May 4, 1998, Special Report. " There Are Two Sides to this Coin »)

Hence the need to take EU enlargement in stages...Slowcoaches should not be allowed to hold back the nimbler-footed...Countries that are most desperate for the boost of EU membership will often be those farthest behind in the race. For that reason, finding ways to encourage the laggards is in some ways more urgent than cheering on the front runners... But perhaps the most important thing is to make this time's also-rans feel wanted. The path to membership should be clearly marked... For more than 30 years, Turkey has been promised that if it manages to get through the same hoops and over the same hurdles that others do, it can join... ( The Economist, July 19, 1997, « Welcome to Europe »)

\section{Construction}

Achieve all that and Helmut Kohl will claim his place in history : chief architect of what he calls « The Europe House »... Given that the Union already costs the average German twice as much as a Dutchman and four times as much as a Briton, and given that the opposition is already suggesting the euro be delayed, perhaps Europe's architect will learn a familiar 
lesson : stable buildings cannot be run up in a hurry. (The Economist, December 23, 1996, «Single-Currency Minded »)

Just as the project for the 1992 single market was the glue for the EC's last piece of constitution-building, the Single European Act of 1986, so the programme leading to a single currency was the chassis that stiffened the Maastricht treaty's improbable bodywork, designed with German precision, unbendable... (The Economist, February 13, 1993, « The End of Never-Never Land »)

\section{Combat}

The euro, he [Mr Kohl] believes, will be the magic weapon that neutralizes his political opponents. (Newsweek, February 2, 1998, « The Global Economy. Once More Around the Track »)

\section{Annexe 3}

Hurricane Maastricht hit Europe a week earlier than expected and with a roar that all but drowned out France's fateful vote on European integration. In its wake lay a twisted political and economic landscape that may take years to recover and may not ever be the same again. Battered as never before in its 13-year history, the European Monetary System will need extensive repairs if it is to serve as a cornerstone of some future monetary union... Britain, where ratification of the treaty on European unity had once been a foregone conclusion, emerged from the tempest in a shaken and vengeful mood and facing a political crisis. This week, Europeans will begin assessing the damage and picking up the pieces. They will also pray that what they experienced was the storm before the calm. But nobody is expecting the good ship Europa to reach safe haven anytime soon. Like many a natural catastrophe, Europe's monetary storm blew up with little warning and left no time to seek shelter ; in fact, it followed a deceptive burst of sunshine... All hurricanes eventually blow themselves out, and the one that hit Europe last week will do so as well. Even its victims will recover : battered as it was, the EMS will continue to limp ahead... But tempests have a way of testing the soundness of structures, and Hurricane Maastricht has exposed for all to see how unprepared the EC is to go forward with monetary and political union. The good ship Europa remains afloat but if it is to begin making headway again, it will need a crew that is prepared to work together - and perhaps even a new chart to guide it to a safe haven in a more united Europe. (“An Ill Wind”, Adam Zagorin, Time, September 28, 1992)

\section{RÉSUMÉS}

Partant du constat que la langue de l'économie contient bon nombre de métaphores, nous nous sommes attachée à étudier plus particulièrement le domaine des monnaies afin de rendre compte de la spécificité du discours sur l'euro et d'affiner une analyse ébauchée à l'occasion d'une approche de la néologie autour de la monnaie unique. Un rappel des notions fondamentales concernant la métaphore en général nous a semblé indispensable pour mieux situer le cadre de notre étude : les questions de sémiotique et de sémantique, de lexicalisation de la métaphore et de métaphore d'invention, ainsi que les différentes fonctions de la métaphore sont abordées. Puis, nous avons passé en revue les registres métaphoriques le plus fréquemment utilisés pour parler des monnaies en général, avant d'examiner le cas de l'euro, et d'essayer d'en faire ressortir la spécificité, en insistant sur les domaines d'emprunt qui caractérisent son discours. Enfin, nous avons tenté d'analyser ce discours et de montrer comment les métaphores peuvent se révéler 
source précieuse de renseignements sur une période donnée et comment le message qu'elles véhiculent peut devenir instructif quant aux opinions de ses auteurs et de ses destinataires.

Since it is commonly acknowledged that the language of economics is studded with metaphors, we have focused our research on the field of currencies and more particularly on the euro, with a view to underlining the specificity of its discourse and to sharpening the analysis we merely outlined in a recent study of neology about the single currency. We felt it necessary to offer a brief review of the fundamental notions about metaphors in order to set the framework of our investigation. The questions of semiotics vs semantics, of dead and living metaphors and of the various functions of metaphors have been raised. Then we have analysed the most frequently used metaphors when dealing with currencies in general, and with the euro in particular, in order to bring out the specificity of the euro and its discourse. Lastly, we have tried to analyse this metaphorical discourse and to show what a precious source of information metaphors can be as regards a given period of time and how telling a message they can convey as to the opinions of both the sender and the receiver.

\section{INDEX}

Keywords : currency, discourse analysis, euro, metaphor

Mots-clés : analyse du discours, euro, métaphore, monnaie

\section{AUTEUR}

\section{CATHERINE RESCHE}

Catherine Resche est maître de conférences en anglais économique, chargée plus particulièrement de l'enseignement de l'anglais financier en DEA Monnaie-Finance-Banque et en DESS Techniques Bancaires et Financières. Elle fait partie du Centre de recherche en langues et cultures (CERLAC) de l'Université Paris 1. Elle s'intéresse à tous les aspects de la langue de spécialité : approche de la lecture, terminologie, phraséologie, dictons, néologie, métaphores. catherineresche@club-internet.fr 